\title{
Quality specifications for roadway bridges. Standardization at a European level
}

\author{
Joan R. Casas \\ UPC-BarcelonaTech, Barcelona, Spain \\ José C. Matos \\ University of Minho, Guimaraes, Portugal
}

\begin{abstract}
Across Europe, the need to manage roadway bridges efficiently led to the development of multiple management systems in different countries. Despite presenting similar architectural frameworks, there are relevant differences among them regarding condition assessment procedures, performance goals and others. Therefore, although existing a complete freedom of traffic between countries, this dissimilarity constitutes a divergent mechanism that has direct interference in the decision making process leading to considerable variations in the quality of roadway bridges from country to country. The need for harmonization is evident. Action TU1406, funded by COST (European Cooperation in Science and Technology), aims to institute a standardized roadway bridges condition assessment procedure as well as common quality specifications (performance goals). Such purpose requires the establishment of recommendations for the quantification of performance indicators, the definition of performance goals and a guideline for the standardization of quality control plans for bridges. By developing new approaches to quantify and assess bridge performance, as well as quality specifications to assure expected performance levels, bridge management strategies will be significantly improved and harmonized, enhancing asset management of ageing structures in Europe. The work developed and the final results and conclusions achieved by COST Action TU1406 will be presented.
\end{abstract}

\section{INTRODUCTION AND BACKGROUND}

Infrastructure managers are facing now conflicting requirements to improve the availability and serviceability of aging infrastructure, while the maintenance planning is constrained by budget restrictions. Many research efforts are ongoing, for the last few decades, ranging from development of bridge management systems, optimization models, life cycle cost analysis, to big data analysis and implementation of artificial intelligence models into decision support tools. Since transport infrastructure is presenting crucial factor for the economy and societal development, it is not only subject to technical requirements, but it is also required to keep up with societal and economic requirements.

In the past years, significant worldwide research has been done regarding condition assessment of roadway bridges. Obtained values from visual inspections and/or non-destructive testing, which provide information regarding the assessed bridge condition state, are compared with previously established goals. As a result, there are currently several methodologies to assess the bridge condition. More recently, the concept of performance indicators (PI) was introduced, simplifying the communication between consultants, operators and owners. However, large deviations continue to exist on how these indicators are defined and obtained in different countries and, therefore, specifications are required for a standardization of this procedure. For a standardized procedure, for example, quality control plans (QCP) are further important instruments which are based on the previously mentioned PIs and pre-specified Performance Goals (PG). However, these PG are difficult to define, as they are highly subjective as they reflect the user and societal requirements. QCP and performance goals are normally stored in the respective 
Bridge Management Systems (BMS). In Europe, most countries have developed their own BMS in recent years as presented in figure 1. Looking to figure 1, it becomes evident an important contradiction. In fact, the different BMS reflect different performance indicators and performance goals among countries. However, do not exist highway traffic restrictions of people or goods between them. The user, travelling across countries may find substantial different quality levels in the bridges he crosses. Therefore, the need for some kind of standardization becomes evident at an European level. As a result, recently, COST (European Cooperation in Science and Technology) Action TU1406 emerged with the main ambition of developing a guideline for the establishment of common QCPs in roadway bridges in Europe, by integrating the most recent knowledge on performance assessment procedures with the adoption of specific goals (Casas, 2016 a,b, Matos et al., 2016). By developing new approaches to quantify and assess bridge performance, as well as quality specifications to assure expected performance levels, it is expected that bridge management strategies will be significantly improved and homogeneized all across Europe, enhancing asset management of aging structures in Europe. . The aim of COST Action TU1406 was to agree between all countries in Europe about common performance goals and quality levels that could be implemented all across Europe

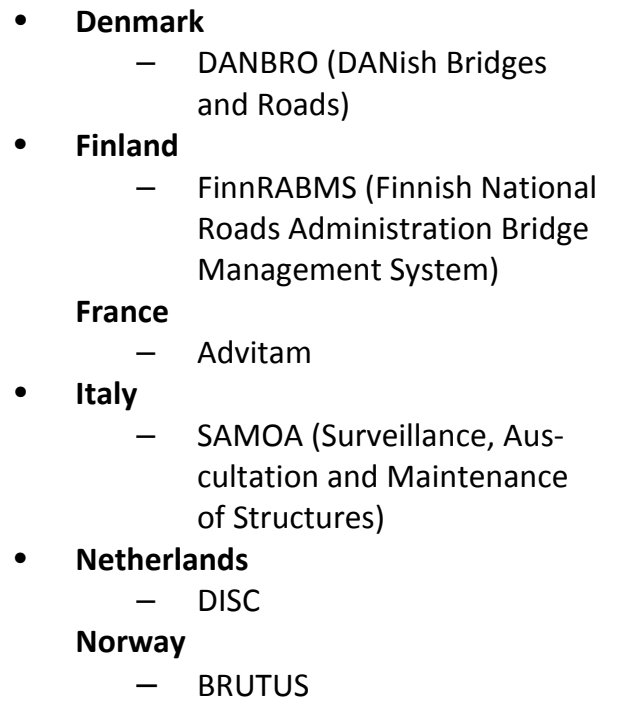

- Sweden

- $\quad$ BMS

- KUBA

- United Kingdom

- STEG (Structures REGister);

- HiSMIS (Highway Structures Management Information System)

- SMIS (Structures Management Information System)

- BRIDGEMAN (BRIDGE MANagement system)

- COSMOS (Computerized System for the Management Of Structures)

Figure 1. Bridge Management Systems in some European countries.

\section{OBJECTIVES OF THE ACTION}

As mentioned before, there is an increasing need of standardization of the quality control plans for highway bridges in Europe. As a response, the overall intention of the Action was to develop a guideline for the establishment of Quality Control (QC) plans in roadway bridges reachable by pursuing the following 5 objectives:

1.- Systematize knowledge on QC plans for bridges in European countries, which will help to achieve a state-of-art report that includes performance indicators and respective goals

2.- Collect and contribute to up-to-date knowledge on performance indicators, including technical, environmental, economic and social indicators

3.- Establish a wide set of quality specifications through the definition of performance goals, aiming to assure an expected performance level

4.- Develop detailed examples for practicing engineers on the assessment of performance indicators as well as in the establishment of performance goals, to be integrated in the developed guideline 
5.- Create a database from COST countries with performance indicator values and respective goals, that can be useful for future purposes.

The number of participants in the action can be seen in figure 2. A total of 264 people has participated, with a high number of Early Stage Researches (ESR). A total of 56 countries worldwide (not only European) participated in the project with a geographical distribution as presented in figure 3 . Between the participants a very representative mix of academics, consultants and bridge owners were present. An Industrial Advisory Board (IAB) with 3 bridge owners and 2 consultants was created in order to give the maximum decision level to those that have to latter apply the recommendations. Also international observers ( 1 per continent) were endorsed in order to compare the results of the Action with worldwide actual state of the art on the subject.

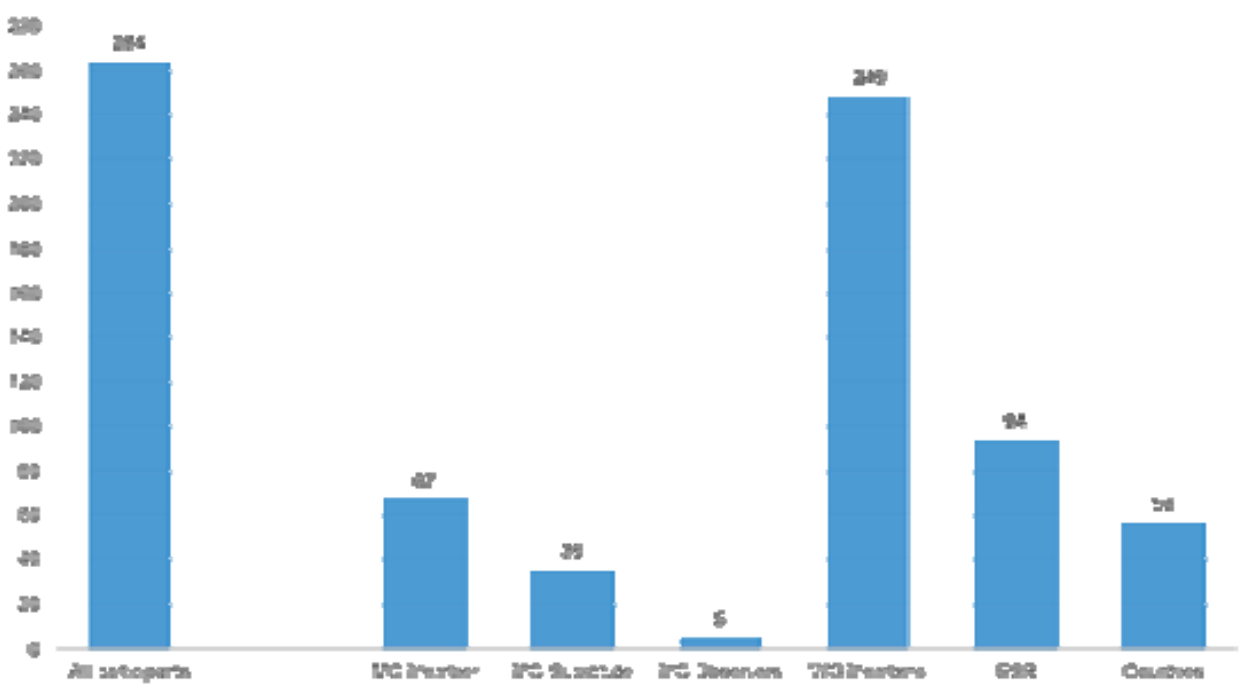

Figure 2. People involved in the Action

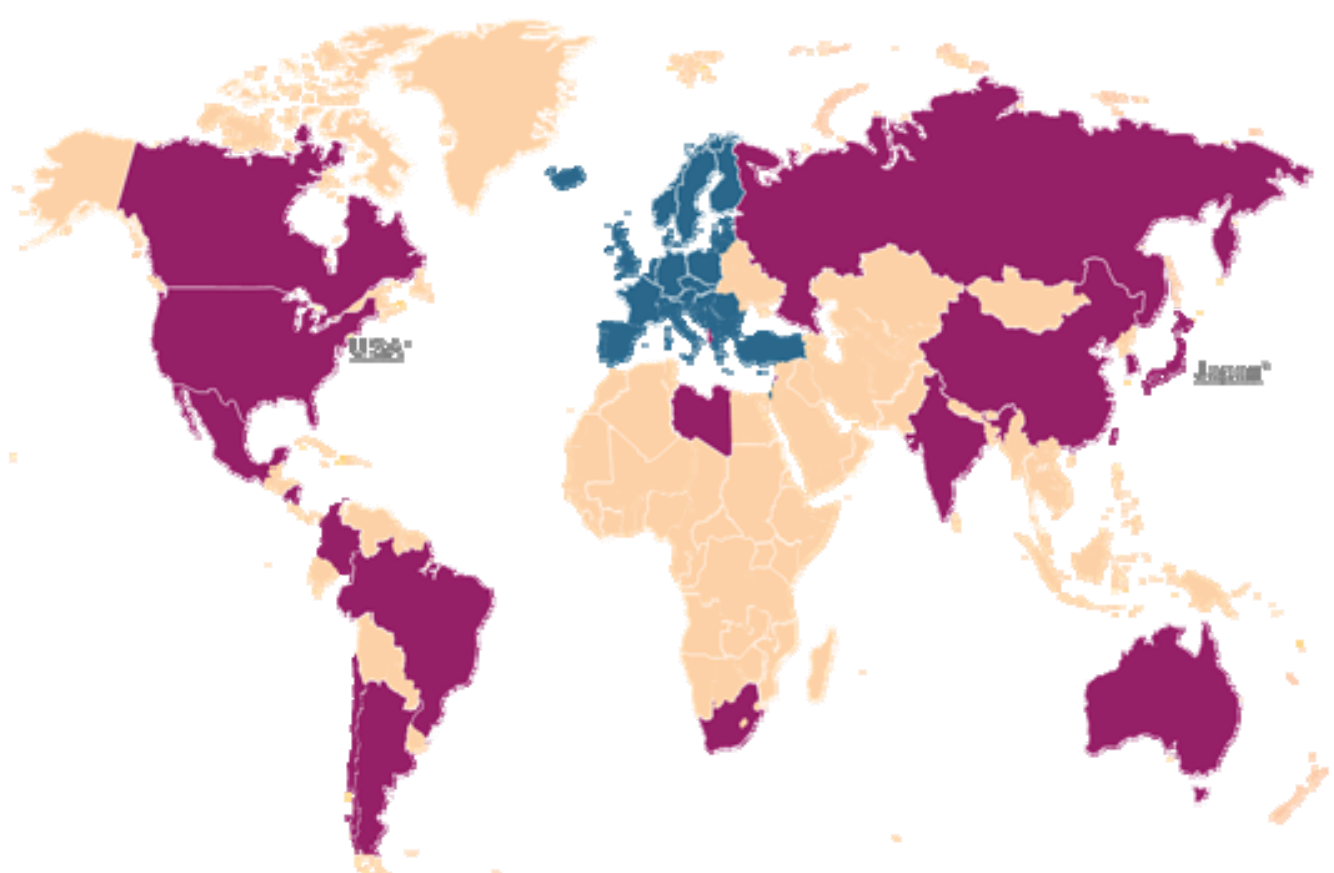

Figure 3. Participating countries. Blue:COST Countries, Red: International Partner Countries. 


\section{DESCRIPTION OF INITIAL WORKS}

\subsection{State-of-the art on QC plans in Europe}

As a starting point it was decided to start looking to the available guidelines and documents related to inspection and maintenance today in use by the bridge/highway owners and operators in Europe. The reason for such decision looks quite evident:

1.- In most countries the performance of bridges is good. Therefore, the agencies, at least in these countries are doing a good job.

2.- We need to know exactly what are they doing in order to improve and enhance (if required) their procedures and rules.

3.- The implementation of a common methodology across Europe with flexibility to accommodate country-specific requirements needs to know what is being done now. If too many changes are proposed, reluctance of bridge owners and operators to perform those changes in the daily operation will appear. In addition, the new harmonized methodology can not disregard all the knowledge accumulated by the owners/operators along many years of bridge inspection and maintenance.

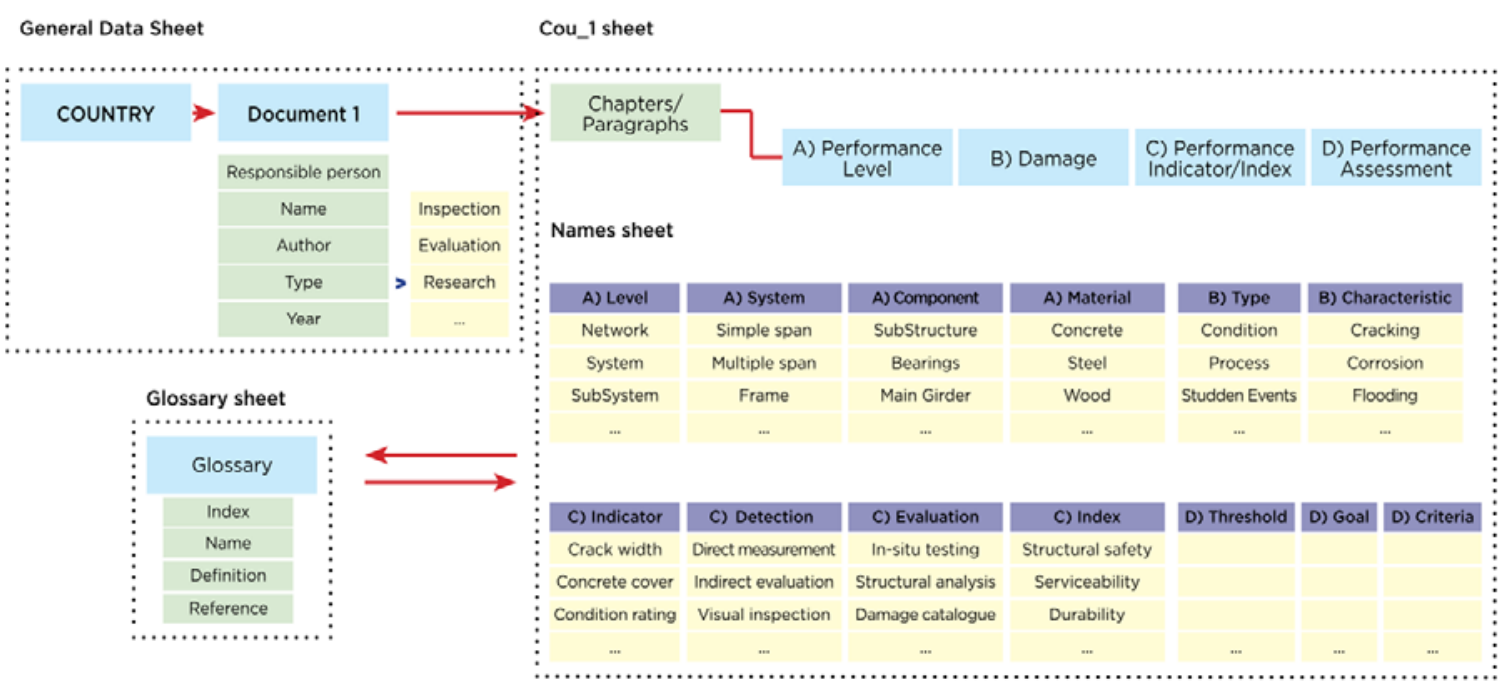

Figure 4. Structure of the Performance indicators database for the survey process

Through the WG1 activities, the development of a PIs database had been defined as an essential component of COST TU1406. The preliminary works developed into the working group indicated that an extended systematic screening on practical national inspection and evaluation documents was necessary in order to obtain a consistent and conclusive information associated with PIs, PGs and performance thresholds (PTs) from each COST partner country. The core of the survey process for obtaining PIs and corresponding key performance indicators (KPI) is given in Figure 4.

Taking into account the high number of languages spoken across Europe, it was obvious that language problems will arise as the required documents from the different owners and operators around Europe are written in many different languages. In many cases, a full code or guideline only presents some pages devoted to the subject of interest (performance indicator, performance goal, quality control, maintenance scenarios,...). Therefore it was seen as unnecessary to translate the whole document. On the contrary, the adopted strategy was to identify a participant in the Action as responsible to collect the relevant parts for the questionnaire of the existing guideline in his country and translate them to English. Of course, the responsible person should be 
somebody with good knowledge and expertise on inspection/assessment of existing bridges in order to identify the relevant parts. Apart from the language problems related to the translation of the documents, another language problem relates to the definition of several terms, as many times the same operation or concept has different English translations or wording ( see for instance the concept of condition state where different terms and words are used in different countries to refer to the same idea). For this reason, a glossary of terms was also developed and agreed among the COST participating countries.

The two COST nominated persons per country, together with the infrastructure operators and owners chose beforehand the relevant documents (e.g. inspection, evaluation, research etc.) from which the PIs, and related information were extracted. Additional information on the survey process, the harmonization of the obtained information, as well as the final data-base of performance indicators and glossary of terms elaborated is fully available in WG1 report of the Action, reachable at www.tu1406.eu

\subsection{Proposed performance goals and KPI}

The second step was to provide an overview of existing performance goals for the indicators previously identified in WG1 and to develop technical recommendations which will specify the performance goals. These goals will vary according to technical, environmental, economic and social factors. As presented in figure 5, the final adopted performance goals were based on the requirements of safety to the user and minimization of cost (including owner, user and environmental costs). As a result, the following KPI (Key Performance Indicators) were decided: Reliability (safety in the structural sense), Safetyy, Availability and Cost. The first two are obtained as an static snapshot of the actual bridge condition, whereas the other two depend on the alternative maintenance scenarios adopted (see figure 5) and therefore become dynamic along time.

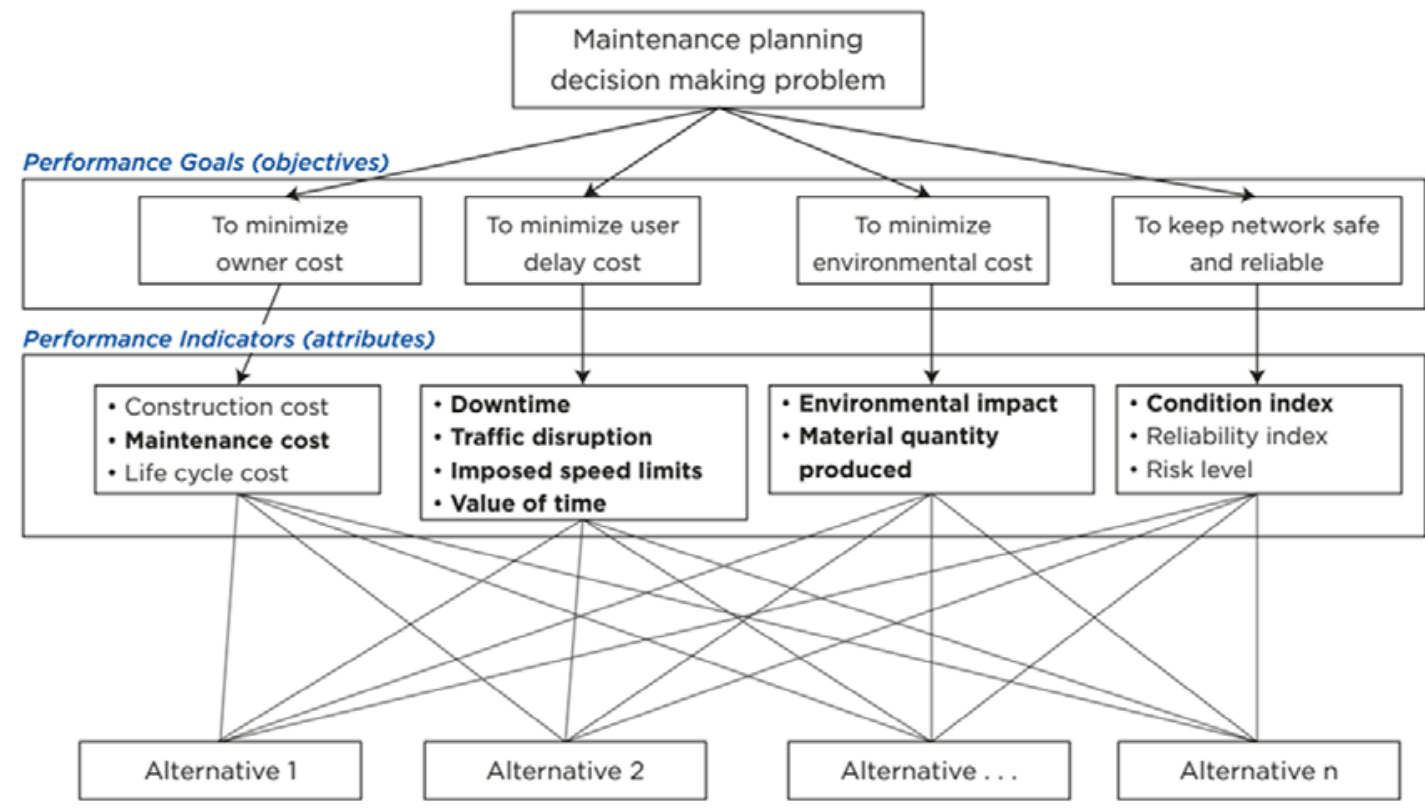

Figure 5. Performance Goals (KPI) and corresponding performance indicators

As some times PGs could be contradictory (increasing of safety also derives in increase of cost, for instance), bridge performance goals should be set as a multi-objective system, taking into account different aspects of bridge and network performance. Also, the described performance goals are defined at a system (bridge) level. In Figure 6, the process of the multiple performance goals assessment is presented, also showing how the transition from the system (bridge) to the network level is achieved. 
In order to get the optimum quality control plan, Multi-criteria decision-making (MCDM) provides a systematic approach to combine these inputs with benefit/ cost information and decision-maker or stakeholder views to rank the alternatives. MCDM is used to identify and quantify decision-maker and stakeholder considerations about various (mostly) non-monetary factors in order to compare alternative courses of action. Hierarchy structure for linking multi-objective bridge performance goals, covering most of the previously mentioned aspects with performance indicators is required. Possible result of multi-criteria assessment of different bridge maintenance alternatives represented in a spider diagram as in Figure 7 for a defined point in time, can be finally used for a decision making about the optimal maintenance or design solution.

Alternatively, the multiple performance criteria can be combined into a so-called utility function, in which all the criteria are brought into a single scale. In order to transform the various out into a single (mostly monetary) scale it is necessary to establish weight factor for the individual types of criteria. Some of the weight factors are available in some countries (for example weight factor for traffic delays, noise, injuries etc.). Depending on the selection of criteria, some weight factor may still need to be developed. In WG2 of TU1406 COST Action, a web-based tool was developed to apply the multi-objective optimization. The developed tool has implemented one of the methods of MCDA, namely Multi-Attribute Utility Theory (MAUT) by using the R Utility package (Reichert et al. 2013). The tool as well as further information on the treatment of performance goals within the Action can be found in the Report of WG2 at www.tu1406.eu

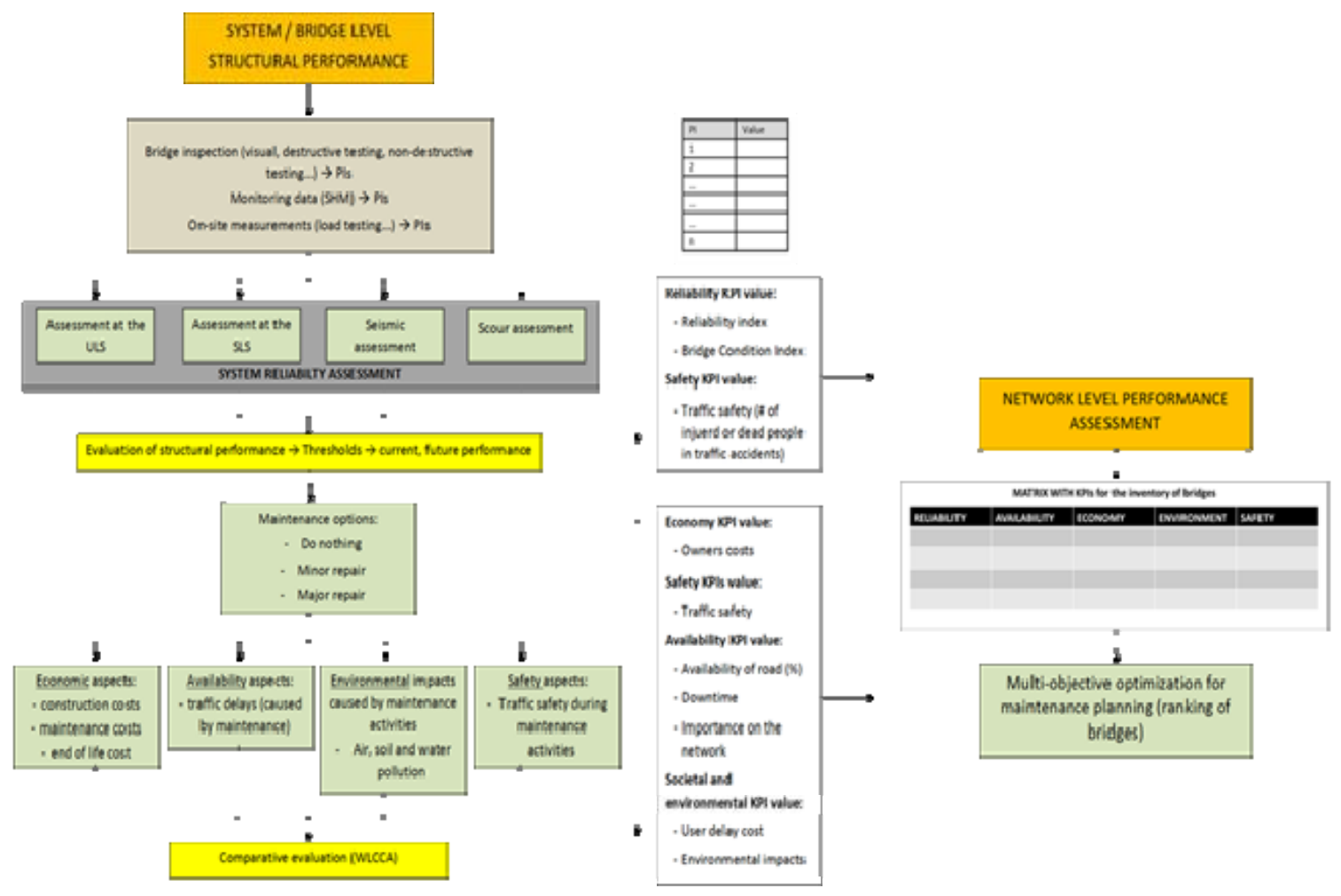

Figure 6. Overview of the process for performance goals assessment and transition from system to network level 


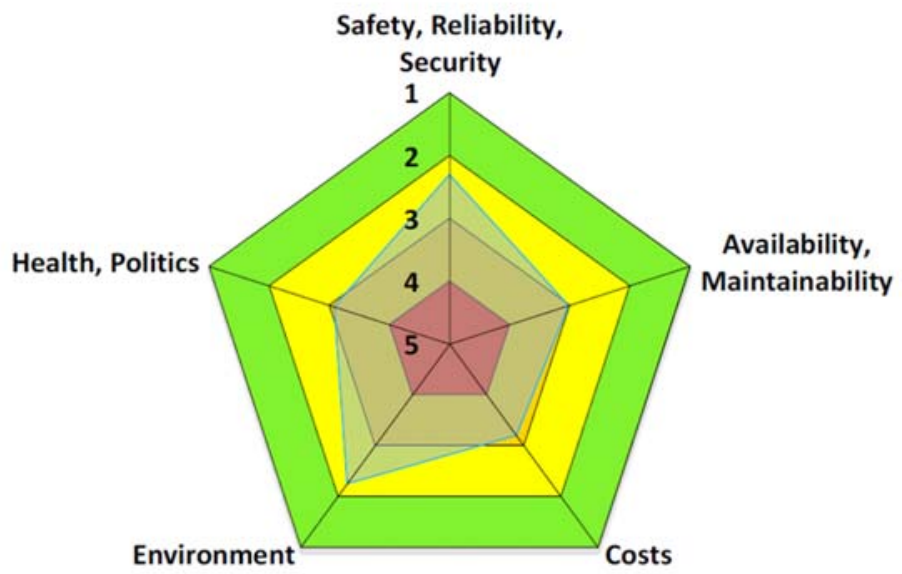

Figure 7. Spider diagram for multi-criteria decision making

\section{FRAMEWORK OF QUALITY CONTROL PLAN}

The quality specification or QC framework aims to provide a methodology with detailed stepby-step explanations for the establishment of QC plans for different bridge types. According to the identified PGs, these plans relate user/society goals, such as:

- Reliability: including the probability of structural failure (structural safety) or operational failure (serviceability)

- Availability: the proportion of time a system is in a functioning condition. In our case is the additional travel time due to imposed traffic regime on the bridge

- Safety (not structural safety): minimize or eliminate people harm during the service life

- Economy: minimize life-cycle cost

- Sustainability: environmental friendliness

In figure 8 is shown the structure that supports the QC plan for highway bridges. Based on the static or dynamic nature of the goals, quality control plans are also divided in 2 groups:

- Static (snap shot) control: to inspect and investigate the bridge and determine whether reliability (structural safety and serviceability) and safety are met. This is fundamentally the basis for the decision making on actions

- Dynamic control: based on the static control and including the plan and actions (maintenance scenarios) to execute in order to ensure the long term fulfilment of safety and serviceability goals. The goals to achieve are related to availability, economy and sustainability as they include the feasible maintenance scenarios that define costs and availability over a certain time frame by using reliability and safety forecasts.

Because the reliability goal should be checked against feasible failures, the practical application of the method has divided the global group of bridges into the following bridge types: frame, arch and continuous beam. For each of these bridge types the most vulnerable zones can be identified and the corresponding PIs related to reliability observed and/or quantified. This division also helps in the process of selecting the best maintenance policy. The goals of availability, economics and sustainability are governed by maintenance scenarios. In fact, the snapshot assessment of availability and costs are of none or little interest. Therefore, the feasible maintenance scenarios (do nothing, preventive and corrective) are defined and the corresponding KPI's evaluated along time according to each maintenance scenario. In this way, the spider diagram of figure 7 is developed along time as presented in figure 9. Regarding the availability indicator, each maintenance intervention requires certain traffic regime, which may include closure for certain type of vehicles or lane closure or narrower lanes. The normal traffic regime can be as- 
signed with the maximum performance value. The other traffic regimes can be ranked by the additional travel time they cause for the road users. This additional travel time can be also monetized according to the guidelines given in the WG2 report. The selected intervention scenario is obtained through MAUT based on the results presented in the diagrams as in figure 9. Further information on the QC framework can be found in the WG3 report at www.tu1406.eu

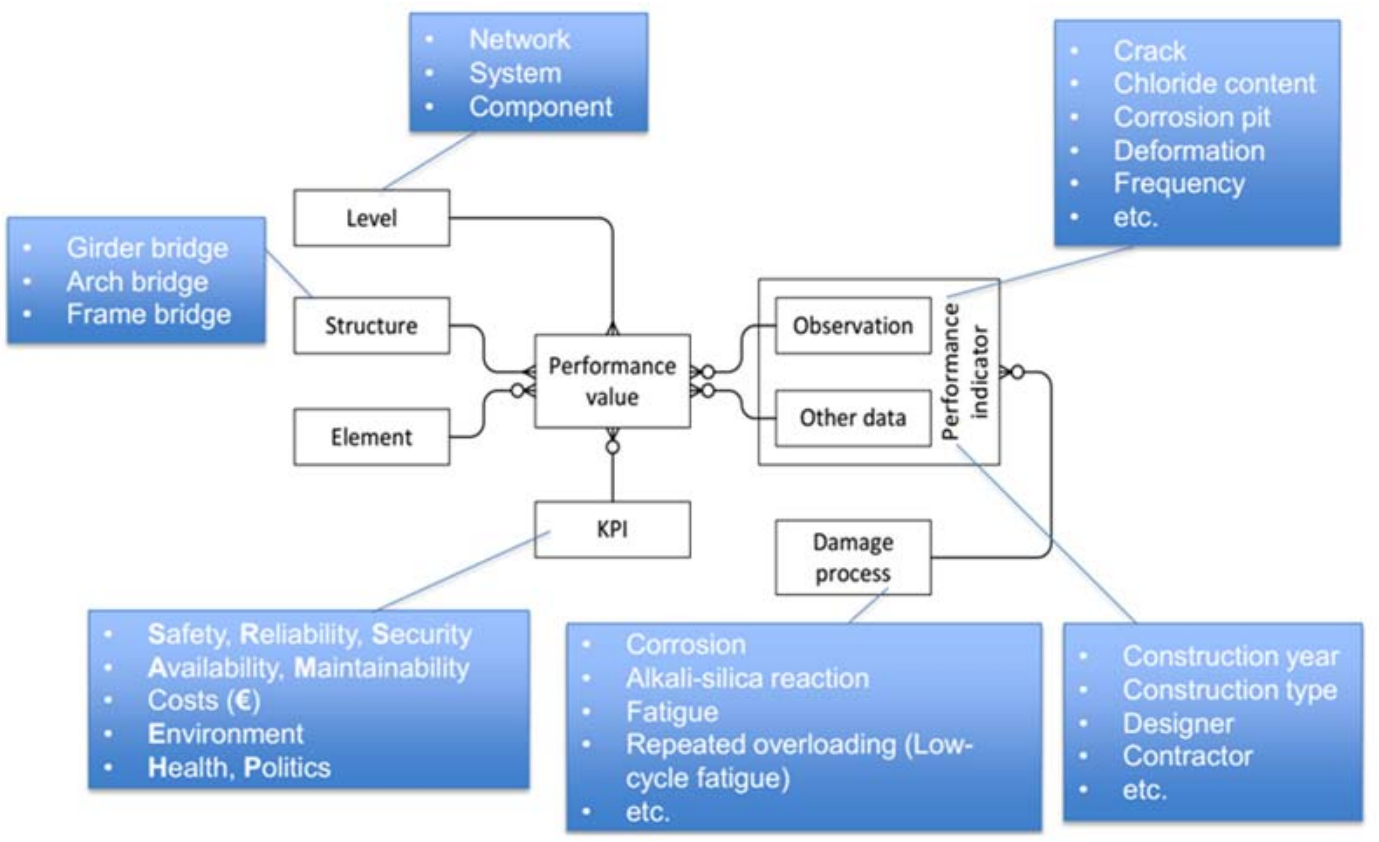

Figure 8. Quality control plan framework
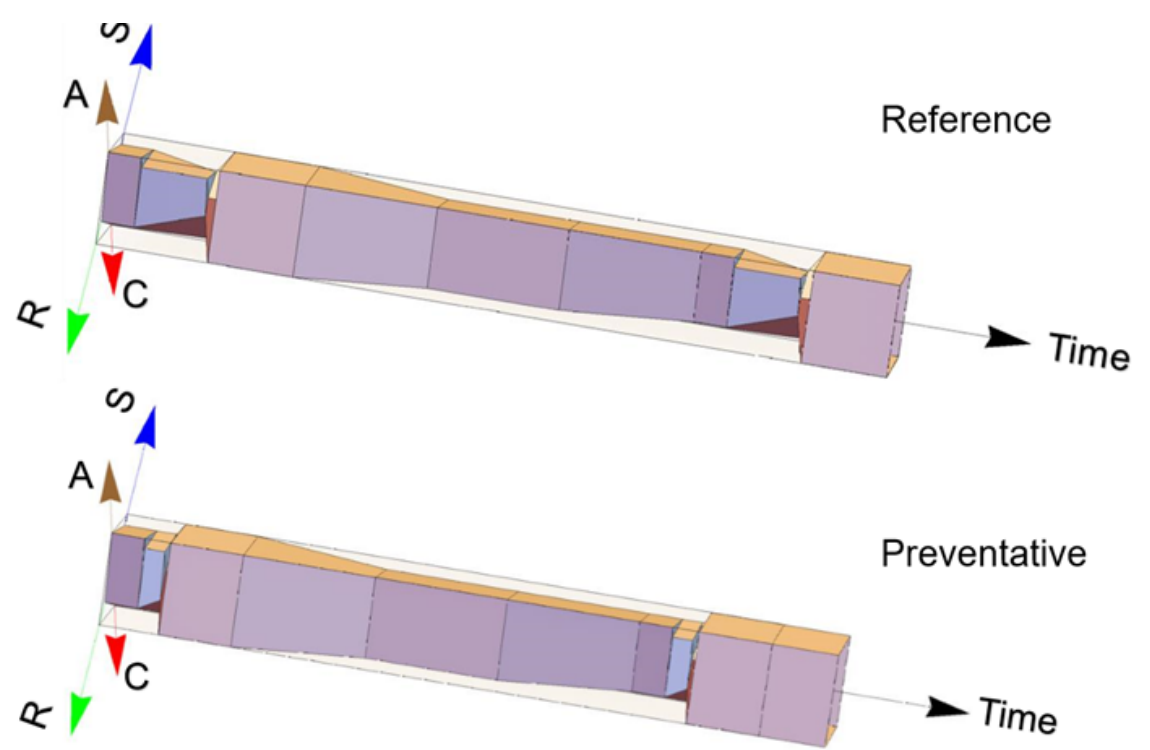

Figure 9. Spider diagram along time for two maintenance scenarios (reference: do nothing and preventative maintenance 


\section{CASE STUDIES}

The case studies developed are grouped according to the bridge types defined in the quality control plan: frame, arch and continuous beam. The proposed framework is thought for standard bridges, most present in the European highways, and not for landmark bridges. As an example, in the frame bridge of figure 10, the vulnerable zones to failure in bending (yellow) and shear (read) are defined (Figure 11) and the corresponding performance indicators related to reliability observed and/or quantified. For instance, regarding a frame type bridge, the regions close to mid-span and supports will be those selected for a reliability analysis. This division also helps in the process of selecting the best maintenance policy. For the frame bridge shown in Figure 10, the corresponding flowchart as proposed in the framework (figure 8) and assigned values of reliability and safety based on the visual inspection is presented in Figure 12. The condition of the bridge observed in the visual inspection is shown in figure 13. It becomes evident in figure 13a the lack of safety due to condition of the bridge edge. Also the state of corrosion in some parts of the bridge (see figure 13b) is used to fix the reliability level. The maximum value for both KPIs is 5 . In this case, the values obtained are 3 for reliability and 2 for safety. In the report of WG4, available at www.tu1406.eu a complete description of 17 case studies, representative of several bridge types and countries, are presented as well as a Guideline with a very detailed flowchart and step-by-step process to apply the quality control framework to a particular bridge (figure 14). This facilitates the task for the bridge engineers and owners to apply the novel approach.

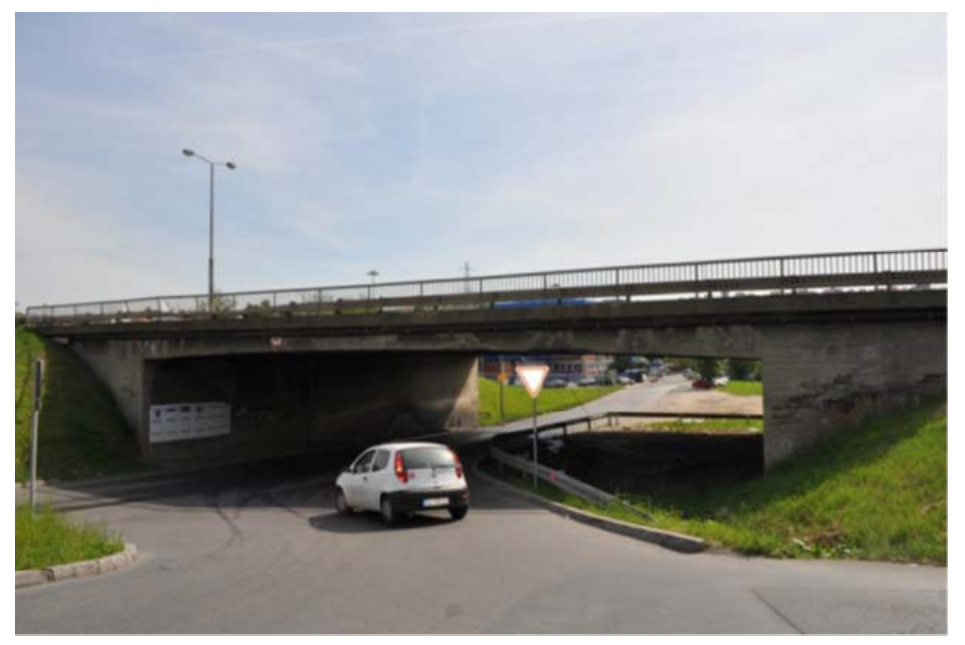

Figure 10. Example: Reinforced concrete frame bridge

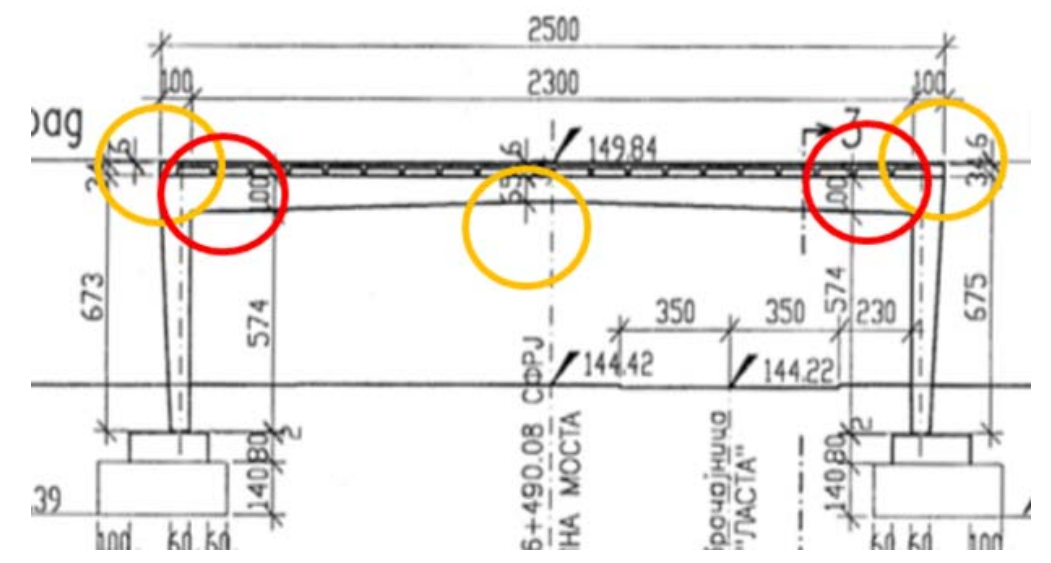

Figure 11. Vulnerable zones in bending and shear 


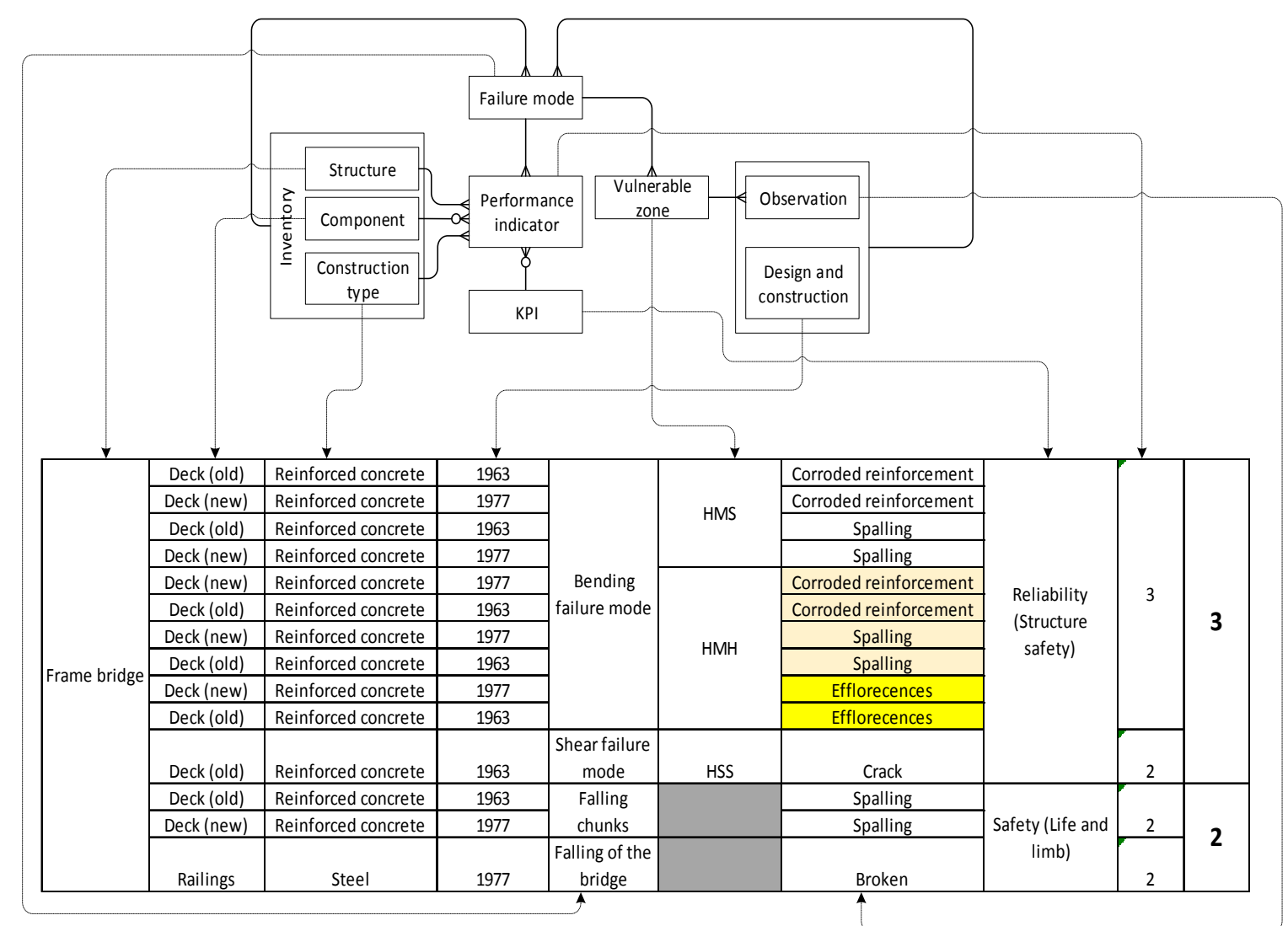

Figure 12. Flowchart and results corresponding to the bridge in figure 10

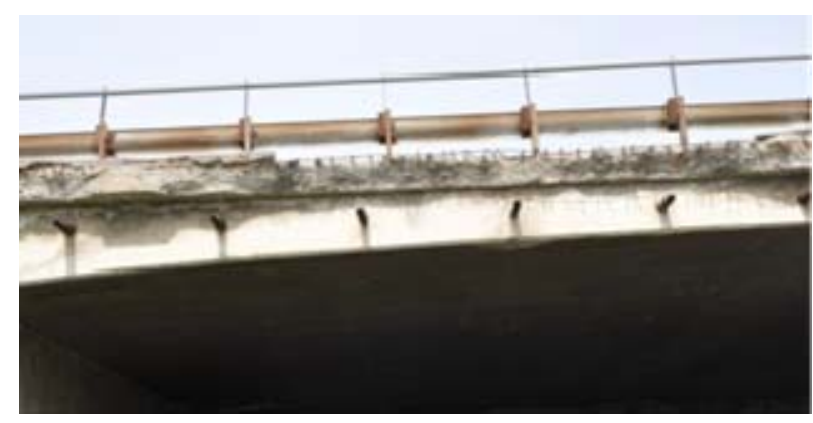

(a)

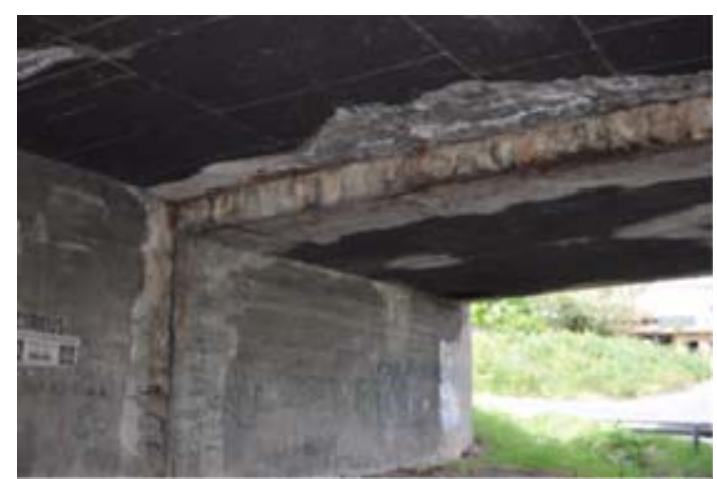

(b)

Figure 13. Condition of the bridge obtained from the visual inspection 


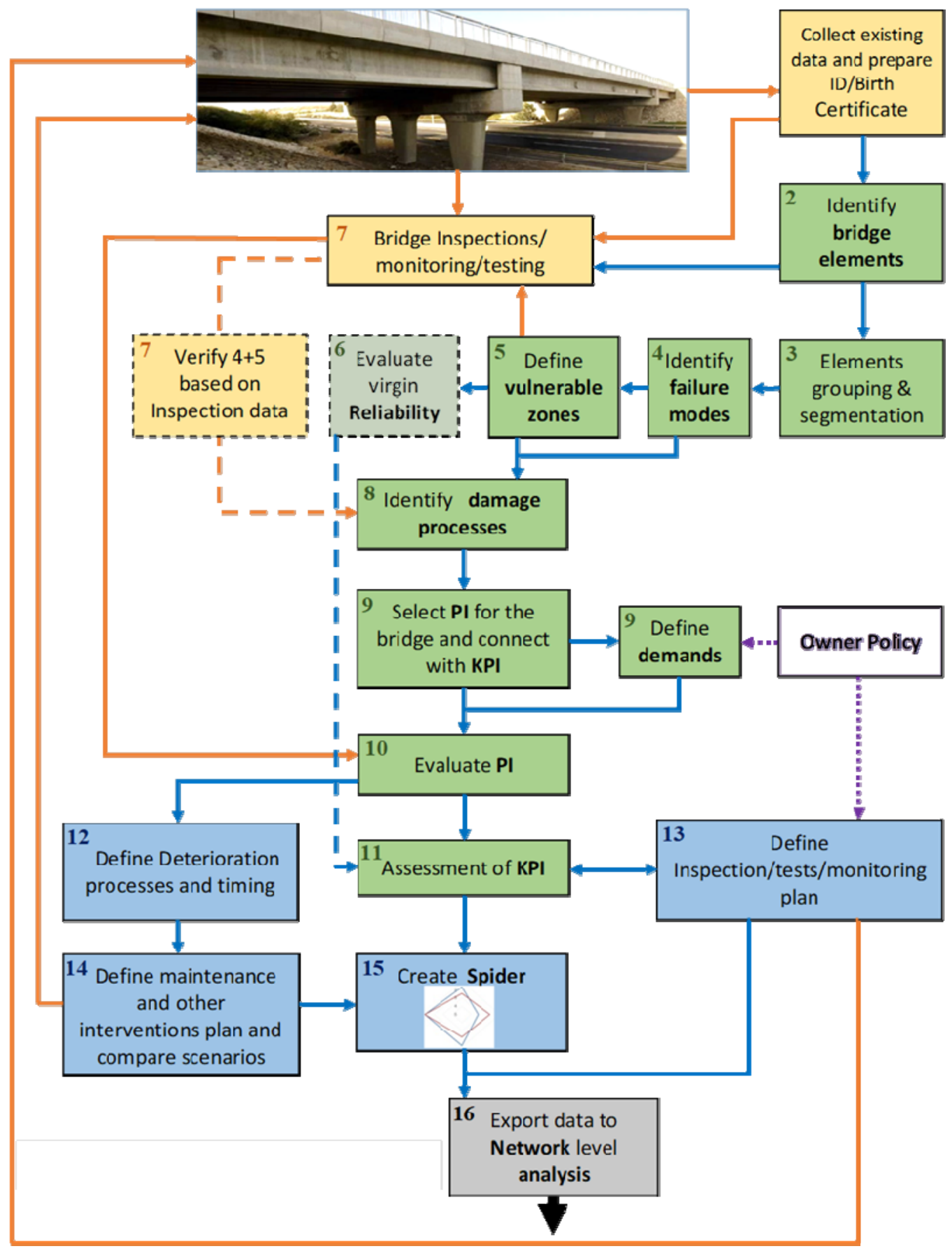

Figure 14. Methodology of Case Study Implementation of Quality Control Process in COST TU1406 


\section{CONCLUSIONS}

During the implementation of asset management strategies, maintenance actions are required in order to keep assets at a desired performance level. In case of roadway bridges, specific performance indicators are established for their components. These indicators can be qualitative or quantitative and can be obtained during principal inspections through a visual examination, nondestructive tests or a temporary or permanent monitoring system. Obtained indicators are compared with performance goals, in order to evaluate if the quality control plan is accomplished. It is verified that there is a large disparity in Europe regarding the way these indicators are quantified and how such goals are specified. Therefore, a discussion at a European networking level through Action COST TU1406, seeking to achieve a standardized approach in this subject, has brought significant benefits. The standardized approach unifies several formats of maintenance management in different networks and countries but allows them to be implemented in the format that they are already operational. Therefore, this Action achieved to bring together, for the first time, both research and practicing community in order to accelerate the establishment of a European guideline in this subject.

After an exhaustive analysis of the data obtained in the survey carried out among European countries, it was concluded that different countries have different definitions of PIs and how they are obtained. In some cases, simple observations are adopted as performance indicators. The methodology of the analysis was based on a deep analysis of the existing bridge management policies and available documents for inspection and evaluation existing in European countries and the main PIs used, with the objective to define a common group of quality specifications and control plans that can be assumed by all these countries. Due to the existence of different interpretations, an additional clustering and homogenization process was required. From this procedure it was possible to verify that all countries have a PI, named condition index, condition rating or deterioration index, mainly obtained through visual inspections. Although in some cases this is the only existing PI used, there are countries, like Denmark or The Netherlands, in which operators and bridge owners are currently using other relevant indicators. In Denmark, concepts like remaining service life, robustness, safety index, reliability and vulnerability are addressed. On the other hand, in The Netherlands, the performance is evaluated by the RAMSSHEEP (reliability, availability, maintainability, safety, security, health, environment, economics and politics) approach, where risk is used to obtain social, environmental, economic and political indicators.

TU1406 has a high societal relevance and brought together a collaborative network of several stakeholders, namely, partners from research and practicing community, aiming to joint efforts to build consensus on this subject. Multidisciplinary and complementary expertise covering a wide range of topics form visual inspection, on-site testing, numerical modelling, asset management and sustainability are considered. The collaborative dialogue developed in the process amongst researchers, engineers and owners and supported through networking, capacity building and training activities in COST TU1406 thus forms an invaluable reference point in the evolution of bridge management in EU.

To establish the suitability of the final reports and proposed methodologies along TU1406 for industrial application, an Industry Advisory Board (IAB) was formed by representatives of bridge owners and consultants. The industry Advisory Board has been asked by the COST TU1406 core group / chair to review the outcome and deliverables from the cost actions and comment based on the applicability in practice for the industry. The IAB uses web-meetings and subsequent individual contributions to arrive at comments and suggested improvements of the reports of relevant WGs. This is especially true where the exploitation of the outcome is relevant for several groups of stakeholders. The IAB has been able to create an impact in various reports, especially commenting on the need for harmonisation of terms and definitions, data screening and access, consolidation of results, feasibility of scope, industry verification, standardization requirements and possibilities, scalability and ease of implementation in an industrial format. 
Based on the results and recommendations of the reports from WG1 to WG4 ( freely available at www.tu1406.eu) and the comments provided by the IAB, normative bodies and stakeholders, COST Action TU1406 has stated the following guidelines in the adoption of a common QC framework for highway bridges in the European countries:

1.- Guideline on the definition of performance indicators for QC and collection of a European data base of observations and performance indicators

2.- Guideline for the adoption of a common QC framework based on the adoption of 4 KPI (Key Performance Indicators: Reliability, Safety, Availability and Cost) and the 3-D spider tool for the practical implementation in an specific bridge in order to obtain the optimum maintenance scenario.

3.- Guideline for practical implementation of the QC plans and definition of the 3-D spider tool for different bridge types.

In the guidelines, a multi-objective approach is recommended to address diverse PGs of a stock of bridges. Five performance aspects (Key Performance Indicators (KPI)) are selected in this regard: 1. Reliability; 2. Availability; 3. Safety; 4. Cost; 5.Environment.

A multi-criteria decision-making (MCDM) approach can systematically combine the inputs with cost-benefit models to rank available decision options about the bridges at component, system or network levels. A web-based Multi-Attribute Utility Theory (MAUT) and the corresponding software tool developed within the Action is recommended to be useful in this regard (https://maut.shinyapps.io/application_of_maut/).

For a simpler approach, the guidelines also recommend the use of a Spider Diagram for QC, by quantifying the overall performance related to the area in the diagram enclosed by KPI values, for a single or a collection of bridges. The KPI is qualitatively quantified between the ordinal scale of 1-5 (1 being the best and 5 being the worst). The most relevant KPIs should be selected for use in a particular situation. When assessed over time, the Spider Diagram forms a tube. The various KPIs can be expressed in their native units and then normalized to obtain their integer values.

Within the QC framework, the KPIs are evaluated for different maintenance scenarios (based on inspection/investigation or prediction), looking for the most feasible one. KPIs of Availability, Economy and Environment can be only reasonably applied as a function of time. Damage processes, defined as independent of combined actions having a detrimental effect on a bridge can be crucial for performance prediction, preventative maintenance and eventual rehabilitation. Information on damage can be obtained from inspection and testing. Impact of natural hazards on bridges is yet to be included in BMS but should be considered to understand consequences.

The QC framework has a a) static and b) dynamic stage. The steps for a static (snapshot) quality control comprise: 1 . Preparatory work (inventory, conceptual weakness of design, material weakness, traffic load changes, identification of vulnerable zones, estimating à priori reliability; 2. Inspection on site (damage detection, material property measurement, sample collection); 3. Laboratory tests; 4. Assessment of the Reliability KPI (resistance reduction estimates, reliability estimates); 5. Assessment of the Safety KPI . The steps for a dynamic quality control comprise:

1. Assessment of remaining service life (damage speed and forecast, time dependent safety and reliability); 2. Maintenance scenario (reference scenario - end of service life, preventative scenario, long term cost, availability and reliability/safety estimates for scenarios); 3. Decision making (multi-objective/attribute optimization, monetize non-monetary KPIs, find optimal scenario).

The following challenges exist for the work carried out:

1.- Training of inspectors are variable from country to country

2.- For preliminary or approximate estimates of reliability, the experience and engineering judgement of the consultant is relied upon and this can have human variability in them.

3.- For damage processes, the assumptions made about the type and rates of changes of damage need to be better calibrated and quantified by inspections and destructive/non-destructive testing.

4.- Definitions around terminology, performance indicators and goals are still not entirely homogenized. 
5.- Understanding of uncertainty and reliability is further required in industrial scenarios.

6.- There needs to be more demonstrations and direct benefits for the owners to implement the more holistic approach considered in this Action.

Finally, the following summary and related ongoing work can be considered:

1.- The Action was extremely successful in a) understanding b) documenting and c) assessing the approaches taken for Road Bridge infrastructure in EU and around the world (e.g. USA, India, Australia, Russia etc.) and collating such information.

2.- Experiences and limitations from bridge owners and managers from various countries were documented

3.- The definitions and deep understanding of key governing terms were better clarified, key performance indicators and their measurements were better established and expanded through the action

4.- For Operational Database, more work is still necessary to identify Key Performance Indicators (KPIs) for achieving Performance Goals for optimal Quality Control Plan and to allocate them with appropriate weights related to their respective levels of importance. The following steps are recommended to select the most important Performance Indicators:

1. Define crucial Performance Goals

2. Categorise Performance indicators in relation to Performance Goals

3. Consider the following qualities for selecting a PI: a) measurability, b) quantifiability, c) availability of target value, d) validity for ranking purposes and e) applicability in making economic decisions

5.- This consorted effort led to an overall approach developed in the Action for assessing road bridges, for which we now have a comprehensive set of case studies using several countries around EU as a demonstration and covering a wide range of road bridges to create a technical evidence base

6.- At the end of the Action we are able to recommend, guidelines for best practice in maintenance and management of Road Bridges. The guidelines will harmonize (note: not homogenize) in EU the principles, approach and methodology based on which their maintenance and management will be carried out while retaining the disparate implementation and levels of data that is currently present in different countries in the presence of resource and funding constraints.

7.- As a follow-up implementation, we have created EUROSTRUCT (www.eurostruct.org) as a first platform for the advancement of such actions through industrial leadership and academic collaborations to provide the bridge managers and owners a platform to discuss, decide and develop further activities in future for safer road bridges.

8.- In order to diffuse the work done by the Action into normative documents we have been liaising closely with relevant bodies (ISO, CEN) to identify and follow-up the scope of contributing to their activities from our results and findings.

\section{ACKNOWLEDGMENTS}

This article is based upon the work from COST Action TU1406: Quality Specifications for Roadway Bridges. Standardization at a European Level, supported by COST (European Cooperation in Science and Technology).

\section{REFERENCES}

Casas, J.R. 2016a. European Standardization of Quality Specifications for Roadway Bridges: an Overview. Proceedings of IABMAS 2016. Foz do Iguaçu (Brasil): Taylor and Francis.

Casas, J.R. 2016b. Quality control plans and performance indicators for highway bridges across Europe. Proceedings of the 5th International Symposium on Lifecycle Civil Engineering. Delft (The Netherlands): Taylor and Francis

Matos, J.C., Casas, J.R., \& Fernandes, S. 2016. COST Action TU1406 Quality Specifications for Roadway Bridges (BridgeSpec). Proceedings of IABMAS-2016, Foz do Iguaçu (Brasil): Taylor and Francis. 
Reichert, P., Schuwirth, N., \& Langhans, S. 2013. Constructing, Evaluating and Visualizing Value and Utility Functions for Decision Support. Environmental Modelling and Software,46:283-91 